Article

\title{
Evaluating the Relationship between Mandibular Third Molar and Mandibular Canal with Semiautomatic Segmentation: A Pilot Study on CBCT Datasets
}

\author{
Rossana Izzetti (D, Marco Nisi, Stefano Gennai *(D) and Filippo Graziani
}

Citation: Izzetti, R.; Nisi, M.; Gennai, S.; Graziani, F. Evaluating the Relationship between Mandibular Third Molar and Mandibular Canal with Semiautomatic Segmentation: A Pilot Study on CBCT Datasets. Appl. Sci. 2022, 12, 502. https://doi.org/ 10.3390/app12010502

Academic Editor: Gabriele Cervino

Received: 16 December 2021

Accepted: 31 December 2021

Published: 5 January 2022

Publisher's Note: MDPI stays neutral with regard to jurisdictional claims in published maps and institutional affiliations.

Copyright: (C) 2022 by the authors. Licensee MDPI, Basel, Switzerland. This article is an open access article distributed under the terms and conditions of the Creative Commons Attribution (CC BY) license (https:// creativecommons.org/licenses/by/ $4.0 /)$.
Unit of Dentistry and Oral Surgery, Department of Surgical, Medical and Molecular Pathology and Critical Care Medicine, University of Pisa, 56126 Pisa, Italy; rossana.izzetti@med.unipi.it (R.I.); marco.nisi@unipi.it (M.N.); filippo.graziani@unipi.it (F.G.)

* Correspondence: stefano.gennai@med.unipi.it

\begin{abstract}
Inferior alveolar nerve injury is the main complication in mandibular third molar surgery. In this context, cone-beam computed tomography (CBCT) has become of crucial importance in evaluating the relationship between mandibular third molar and inferior alveolar nerve. Due to the growing interest in preoperative planning in oral surgery, several post-processing techniques have been implemented to obtain three-dimensional reconstructions of a volume of interest. In the present study, segmentation techniques were retrospectively applied to CBCT images in order to evaluate whether post-processing could offer better visualization of the structures of interest. Forty CBCT examinations performed for inferior third molar impaction were analyzed. Segmentation and volumetric reconstructions were performed. A dataset composed of multiplanar reconstructions for each study case, including segmented images, was submitted for evaluation to two oral surgeons, two general practitioners and four residents in oral surgery. The visualization of root morphology, canal course, and the relationship with mandibular cortical bone on both native CBCT and segmented images were assessed. Inter-rater agreement showed values of intraclass correlation coefficient (ICC) above 0.8 for all the examined parameters. Oral surgeons presented higher ICC values $(p<0.05)$. Segmented images can improve preoperative evaluation of the third molar and its relationship with the surrounding anatomical structures compared to native CBCT images. Further evaluation is needed to validate these preliminary results.
\end{abstract}

Keywords: cone-beam computed tomography; molar; third; oral surgical procedures; diagnostic imaging; image interpretation; computer-assisted

\section{Introduction}

Inferior alveolar nerve injury is one of the major complications following the removal of the mandibular third molar, and still represents an issue in surgical planning [1-3]. Inferior alveolar nerve injury is reported to occur in $0.4-8 \%$ of cases [4]. Depth of impaction and third molar angulation have been claimed to be risk factors related to nerve injury.

Preoperative radiographic assessment is necessary to determine the relative anatomic relationship of the inferior alveolar nerve canal with third molar roots. Although panoramic radiography can be supportive in identifying high risk patients, a three-dimensional (3D) visualization of the anatomical structures of interest is often needed [5].

The introduction of cone-beam computed tomography (CBCT) for the study of highrisk patients has led to an improvement in surgical performance by providing additional information on the nerve position [6,7]. CBCT is becoming increasingly used due to the lower radiation dose to the patient compared to multi-slice $\mathrm{CT}$, the possibility to perform focused examinations with a reduced field of view, and the lower costs [8]. However, CBCT presents limited contrast resolution, which hinders the evaluation of lesions involving both hard and soft tissues [9]. Moreover, it should be borne in mind that CBCT finds indication 
when first level diagnostic imaging techniques, and in particular panoramic radiography, highlight the presence of a close relationship between the inferior third molar and the inferior alveolar nerve canal [10]. The current recommendation is therefore to prescribe CBCT of the mandibular third molar when the surgeon has a very specific clinical question in an individual patient case that cannot be answered by conventional imaging [11].

The topographic relationship between the inferior alveolar nerve canal and the third molar is a well-recognized factor associated with postoperative sensory dysfunction [6]. The direct contact between the nerve and third molar roots, a buccal or lingual position of the nerve, a narrowing of the canal, and the loss of canal cortical bone appear to be associated with higher risk of nerve injury following third molar surgery [6]. Moreover, severe compression of the inferior alveolar canal can result in a significantly increased risk of postoperative inferior alveolar nerve injury [12]. Importantly, when a close relationship between the mandibular third molar and inferior alveolar canal is detected, CBCT imaging appears to significantly reduce the incidence of temporary nerve impairment after third molar removal [4].

In several medical fields, image post-processing of 3D datasets obtained from CBCT studies, and in particular segmentation, has been extensively applied to retrieve further information from diagnostic imaging [13-15]. Segmentation techniques have been predominantly reported for the selective localization and analysis of structures of interest by extracting and labeling groups of voxels of selected density, obtaining relevant information on different anatomical structures and providing a voxel-specific 3D reconstruction [16,17].

The aim of the present work was to assess the potential role of segmentation in the pre-surgical evaluation of the relationship between impacted third molars and inferior alveolar nerve canal.

\section{Materials and Methods}

\subsection{Study Sample}

This retrospective study was conducted on 40 anonymized CBCT examinations performed for the evaluation of mandibular third molar impaction between September 2020 and June 2021. The study was performed on Digital Imaging and Communications in Medicine (DICOM) data retrospectively retrieved from the Radiology Information Systems and Picture Archiving and Communication Systems (RIS/PACS) system and pertaining to male and female patients aged 19-70 years treated for mandibular third molar impaction at the Unit of Dentistry and Oral Surgery, University Hospital of Pisa (Pisa, Italy). The study was conducted following the principles stated in the Declaration of Helsinki.

\section{2. $C B C T$ Segmentation}

Image segmentation was performed using the open source software ITK-SNAP (version 3.6.0, http://www.itksnap.org/, accessed on 30 December 2021) [18] by a single operator expert in oral and maxillofacial radiology. ITK-SNAP allows to combine manual and semiautomatic tools for extracting structures in 3D image data [18]. Prior to beginning the study, pre-training on a sample of 20 datasets which were not part of the study was performed in order to improve intra-operator repeatability. The inferior alveolar nerve, the mandibular third molar, and the buccal and lingual cortex were identified and delineated through semiautomatic segmentation on sagittal, axial, and coronal slices. Subsequently, manual segmentation was performed to assure correct segmentation. Once automatic and manual segmentation were completed, 3D rendering was obtained using 3D MPR Viewer tool (Figures 1-3). 

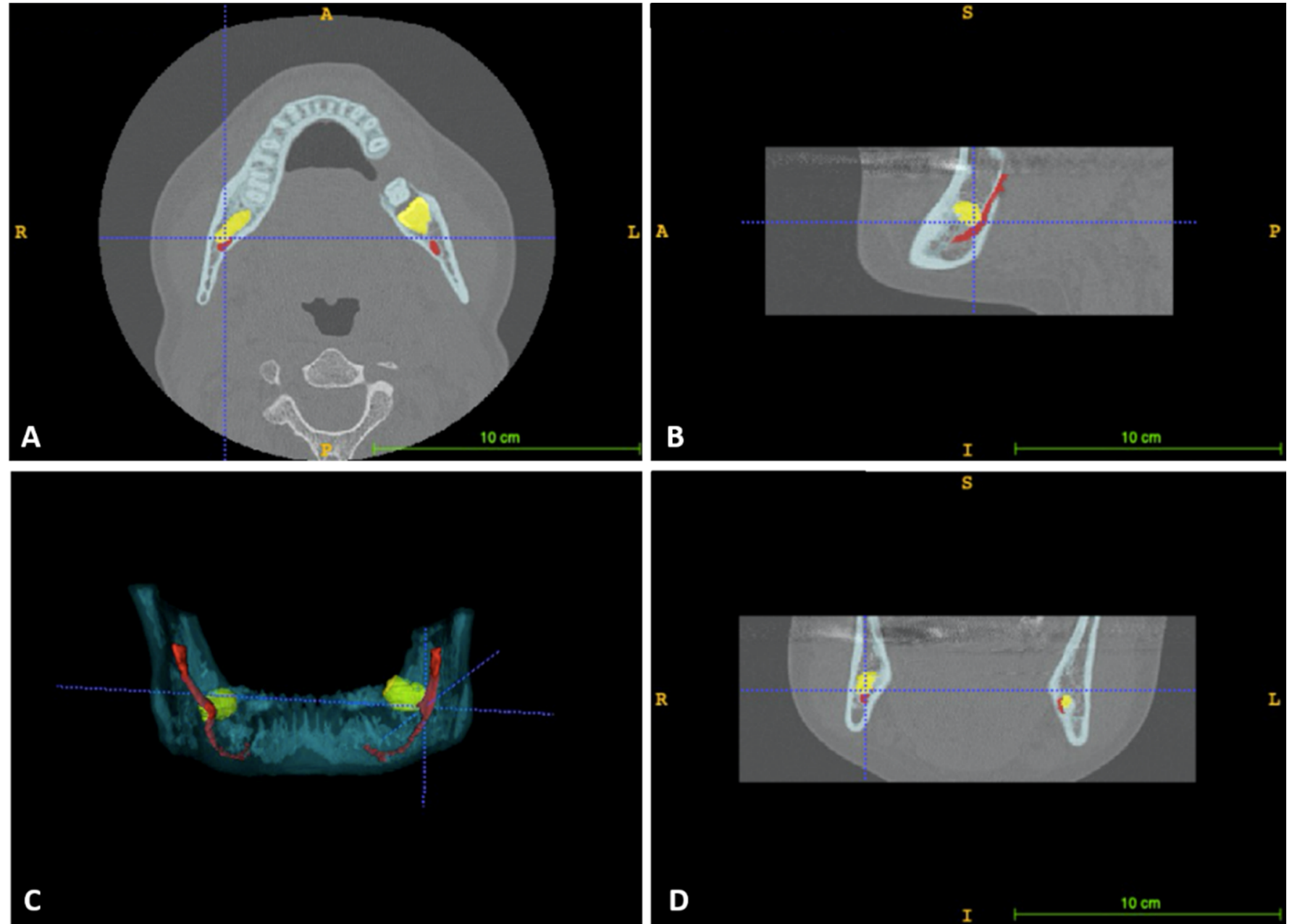

Figure 1. Software interface for image segmentation; in yellow impacted third molars can be seen, while inferior alveolar nerve is marked in red. (A) Axial view; (B) Sagittal view; (C) Segmented volume; (D) Coronal view.

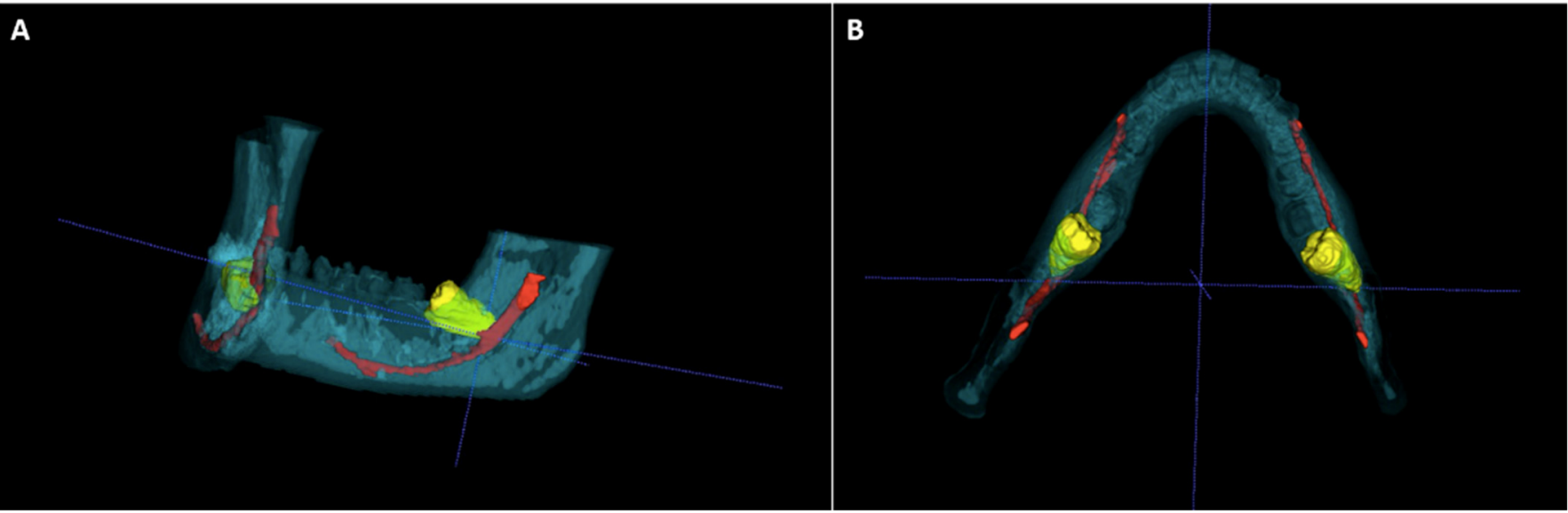

Figure 2. Segmented images. The relationship between bilaterally impacted third molars and inferior alveolar nerve can be observed. (A) 3D reconstruction of the mandible with segmented inferior alveolar nerve (red) and third molars (yellow); (B) Axial view. 


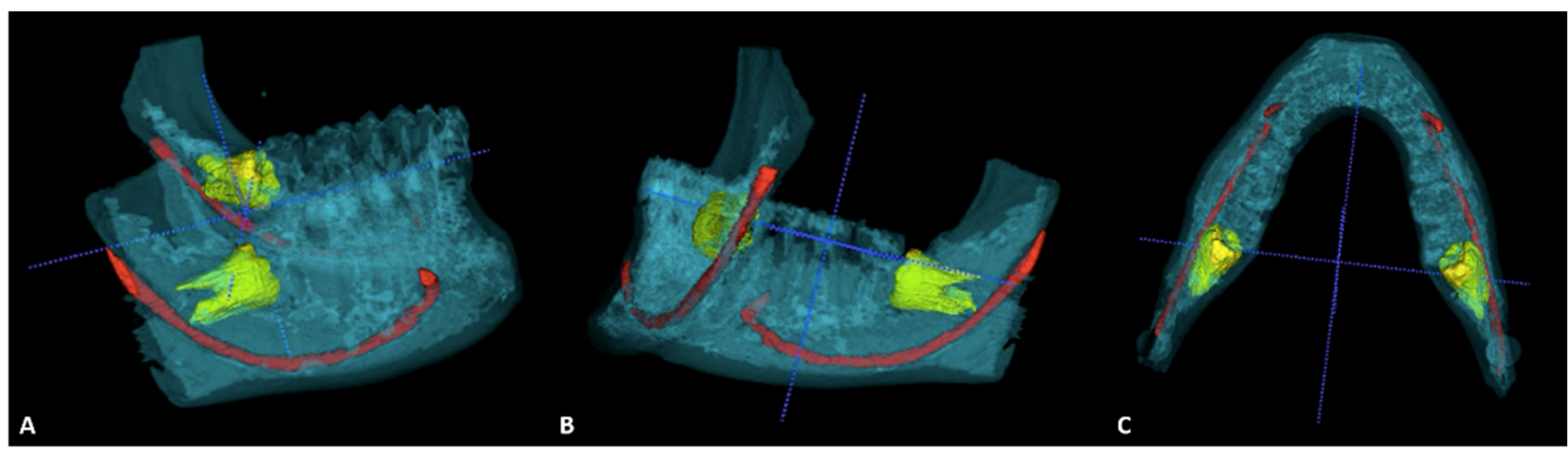

Figure 3. Segmented images. Another case of bilateral impaction is shown. Segmentation can exactly reproduce complex root anatomy and the relationship of impacted third molars with surrounding structures. (A) View of the right third molar; (B) View of the left third molar; (C) Axial view.

\subsection{Image Analysis}

Forty datasets were created. Two oral surgeons, two general practitioners, and four residents in oral surgery separately evaluated the radiologic features of each dataset, which was composed by CBCT and segmented images. Prior to study beginning, a training session was performed to clarify the rating scale and calibrate the readers on the parameters to be assessed. The visualization of root morphology, visualization of canal course, and the relationship with mandibular cortical bone were assessed. Root morphology was classified as: (1) root axis parallel to the major tooth axis; (2) presence of root curvature; (3) bifid apex. Canal course was classified as: (1) absence of relationship with third molar roots; (2) contact between the roots and the inferior alveolar nerve with the preservation of the lamina dura of the canal; (3) close contact between the roots and the inferior alveolar nerve with the loss of the lamina dura of the canal. The relationship of the third molar with mandibular cortical bone was classified as: (1) normal cortical bone; (2) thinning of the cortical bone; and (3) cortical bone fenestration.

\subsection{Statistical Analysis}

Data are expressed as mean and standard deviation (SD). All statistical analyses were conducted using SPSS software (Statistical Package for Social Sciences, version 26.0-SPSS, Chicago, IL, USA). Concordance between readers was assessed with intraclass correlation coefficient (ICC), defined by dividing the variance from the cases by the sum of variances from all sources. Significance was set for $p<0.05$.

\section{Results}

\subsection{Sample Characteristics}

The 40 СBCT datasets belonged to 21 males (52.5\%; mean age 25.36, SD 6.45) and 19 females (47.5\%; mean age 30.11, SD 6.89). The $40 \%$ of mandibular third molars were on the right side, the $60 \%$ on the left side. Bilateral impaction was observed in $27 \%$ of cases. The inclination was $35 \%$ mesial, $20 \%$ distal, $25 \%$ horizontal, and $20 \%$ vertical.

\subsection{Image Analysis}

The inter-rater agreement showed values of intraclass correlation coefficient (ICC) above 0.8 for all the examined parameters when evaluating all the practitioners as a unique sample (Table 1). 
Table 1. Inter-rater agreement evaluated with the intraclass correlation coefficient (ICC) for examiners of all skill levels. Assessment of the root morphology, canal course, and cortical bone through CBCT and segmented images.

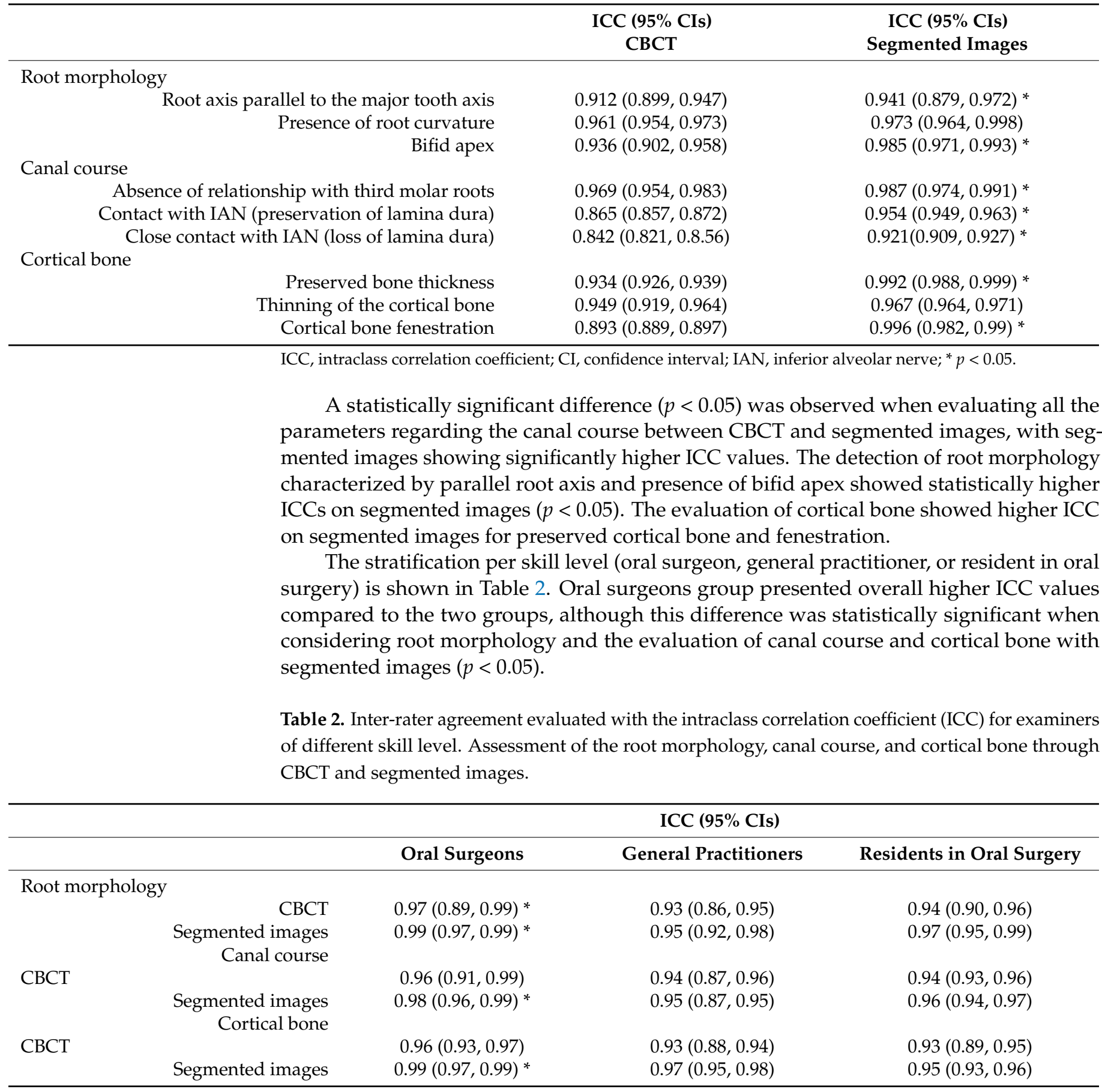

ICC, intraclass correlation coefficient; $\mathrm{CI}$, confidence interval; ${ }^{*} p<0.05$.

\section{Discussion}

Segmented images appear to improve the visualization of root morphology, inferior alveolar nerve canal course and mandibular cortical bone. Higher ICCs were found for segmented images compared to CBCT images. Importantly, the visualization of the anatomical structures of interest improved in all groups regardless of the experience level when evaluating segmented images, thus suggesting that this technique can be supportive in achieving better $\mathrm{CBCT}$ imaging interpretation. 
The use of 3D models has been previously employed for the analysis of various pathological conditions of the oral and maxillofacial region. Botticelli et al. [19] evaluated the effectiveness of 3D models obtained from CBCT datasets in localizing unerupted maxillary canines. According to their results, conventional 2D techniques-including panoramic radiography, lateral cephalogram, and periapical radiographs-appeared less reliable than 3D reconstructions, in particular when evaluating the mesio-distal position of the apex, the bucco-palatal position of the canine, and the overlap with the lateral incisor. Overall, 3D images provided increased precision in the localization of the maxillary canines and improved the estimation of the space conditions in the arch, resulting in a change in diagnosis and treatment planning.

Similarly, Michetti et al. [20] reported a strong correlation in the evaluation of root canal anatomy between 3D post-processed images from CBCT datasets and histologic sections on ex-vivo specimens. In both studies, 3D models provided improved visualization of the structures of interest compared to 2D imaging and native CBCT datasets. However, the use of volumetric reconstructions of the entire CBCT volume was reported. In our study, segmentation techniques were applied in order to obtain selective information on the structures of interest.

Several studies reported on the application of different segmentation techniques. $\mathrm{Li}$ et al. [21] reported the use of a morphological contour interpolation algorithm for the reconstruction of synthetic contours and anatomical structures following guided bone regeneration. Antila et al. [22] segmented the mandible, teeth, maxilla, and zygomatic bones using an algorithm which reached an accuracy of $0.5 \mathrm{~mm}$. Abdolali et al. [23] applied content-based image retrieval for the assessment of maxillofacial lesions. However, when compared to manual segmentation, automated methods appeared slightly less accurate in feature extraction.

Koerich et al. [24] employed standardized threshold segmentation to generate 3D surface models of the maxilla and the mandible with voxel-based superimposition, and reported this method to be fast and accurate.

Shaheen et al. [25] applied segmentation techniques to three different CBCT datasets acquired with different protocols, and found high accuracy for tooth segmentation as compared to anatomical tooth morphology. Another ex-vivo experience was reported by Agbaje et al. [26], who employed mandibular canal tracking to locate and trace the nerve canal in order to improve surgical performance.

Although the variability in the protocols and the limited number of adequate studies hinders the drawing of firm conclusions on the validity and reliability of CBCT-generated 3D models [13], overall it seems that image post-processing may be advantageous in providing additional information, especially in cases of presurgical assessment.

In our study, we employed semiautomatic segmentation as it combines the efficiency and repeatability of automatic segmentation with manual outlining of the region of interest [25]. The use of ITK-SNAP for segmentation has been previously reported and validated, being a highly reliable and efficient alternative to manual tracing [26,27]. With a 3D image-based planning software, tracking of the inferior alveolar nerve canal course can be obtained, creating a virtual replica of the canal, which can give information on shape, curve, direction, and diameter of the nerve.

The present study has some limitations. Firstly, it was retrospectively performed on imaging datasets in the absence of clinical correlation. Secondly, manual segmentation requires training and, although supported by automatic methods, it could require some experience in image post-processing. Nonetheless, these preliminary results suggest that the presurgical evaluation through semiautomatic segmentation of impacted third molars and the surrounding structures may be effective in delineating the relative relationship between third molar roots, inferior alveolar nerve canal, and mandibular cortical bone. 


\section{Conclusions}

Semiautomatic segmentation of $\mathrm{CBCT}$ datasets seems promising in improving the visualization of anatomical structures prior to third molar surgery. Further assessment is needed to fully evaluate the potential role of segmentation techniques in the presurgical planning of complex clinical cases.

Author Contributions: Conceptualization, R.I., M.N. and S.G.; Methodology, S.G.; Software, S.G.; Validation, R.I., M.N., S.G. and F.G.; Formal analysis, R.I. and S.G.; Investigation, R.I. and M.N.; Resources, R.I., M.N., S.G. and F.G.; Data curation, R.I. and S.G.; Writing—original draft preparation, RI., M.N. and S.G.; Writing—review and editing, M.N., S.G. and F.G.; Visualization, R.I., M.N. and S.G.; Supervision, F.G.; Project administration, R.I M.N. and S.G. All authors have read and agreed to the published version of the manuscript.

Funding: This research received no external funding.

Institutional Review Board Statement: The study was conducted according to the guidelines of the Declaration of Helsinki. Ethical review and approval were waived for this study due to the study not involving humans but only anonymized datasets.

Informed Consent Statement: Not applicable.

Data Availability Statement: Data is contained within the article.

Conflicts of Interest: The authors declare no conflict of interest.

\section{References}

1. Ueda, M.; Nakamori, K.; Shiratori, K.; Igarashi, T.; Sasaki, T.; Anbo, N.; Kaneko, T.; Suzuki, N.; Dehari, H.; Sonoda, T.; et al. Clinical significance of computed tomographic assessment and anatomic features of the inferior alveolar canal as risk factors for injury of the inferior alveolar nerve at third molar surgery. J. Oral Maxillofac. Surg. 2012, 70, 514-520. [CrossRef]

2. Hasegawa, T.; Ri, S.; Shigeta, T.; Akashi, M.; Imai, Y.; Kakei, Y.; Shibuya, Y.; Komori, T. Risk factors associated with inferior alveolar nerve injury after extraction of the mandibular third molar-A comparative study of preoperative images by panoramic radiography and computed tomography. Int. J. Oral Maxillofac. Surg. 2013, 42, 843-851. [CrossRef]

3. Qi, W.; Lei, J.; Liu, Y.N.; Li, J.N.; Pan, J.; Yu, G.Y. Evaluating the risk of post-extraction inferior alveolar nerve injury through the relative position of the lower third molar root and inferior alveolar canal. Int. J. Oral Maxillofac. Surg. 2019, 48, 1577-1583. [CrossRef] [PubMed]

4. Korkmaz, Y.T.; Kayıpmaz, S.; Senel, F.C.; Atasoy, K.T.; Gumrukcu, Z. Does additional cone beam computed tomography decrease the risk of inferior alveolar nerve injury in high-risk cases undergoing third molar surgery? Does CBCT decrease the risk of IAN injury? Int. J. Oral Maxillofac. Surg. 2017, 46, 628-635. [CrossRef]

5. Borgonovo, A.E.; Rigaldo, F.; Maiorana, C.; Grossi, G.B.; Augusti, D.; Re, D. CBCT evaluation of the tridimensional relationship between impacted lower third molar and the inferior alveolar nerve position. Minerva Stomatol. 2017, 66, 9-19. [CrossRef] [PubMed]

6. Wang, D.; Lin, T.; Wang, Y.; Sun, C.; Yang, L.; Jiang, H.; Cheng, J. Radiographic features of anatomic relationship between impacted third molar and inferior alveolar canal on coronal CBCT images: Risk factors for nerve injury after tooth extraction. Arch. Med. Sci. 2018, 14, 532-540. [CrossRef] [PubMed]

7. Haas, L.F.; Dutra, K.; Porporatti, A.L.; Mezzomo, L.A.; De Luca Canto, G.; Flores-Mir, C.; Corrêa, M. Anatomical variations of mandibular canal detected by panoramic radiography and CT: A systematic review and meta-analysis. Dentomaxillofac. Radiol. 2016, 45, 20150310. [CrossRef] [PubMed]

8. Izzetti, R.; Gaeta, R.; Caramella, D.; Giuffra, V. Cone-Beam Computed Tomography vs. Multi-Slice Computed Tomography in paleoimaging: Where we stand. Homo 2020, 71, 63-72. [CrossRef]

9. Izzetti, R.; Vitali, S.; Gabriele, M.; Caramella, D. Feasibility of a combination of intraoral UHFUS and CBCT in the study of peri-implantitis. Oral Surg. Oral Med. Oral Pathol. Oral Radiol. 2019, 127, e89-e94. [CrossRef] [PubMed]

10. Izzetti, R.; Nisi, M.; Aringhieri, G.; Crocetti, L.; Graziani, F.; Nardi, C. Basic Knowledge and New Advances in Panoramic Radiography Imaging Techniques: A Narrative Review on What Dentists and Radiologists Should Know. Appl. Sci. 2021, 11, 7858. [CrossRef]

11. Del Lhano, N.C.; Ribeiro, R.A.; Martins, C.C.; Assis, N.; Devito, K.L. Panoramic versus CBCT used to reduce inferior alveolar nerve paresthesia after third molar extractions: A systematic review and meta-analysis. Dentomaxillofac. Radiol. 2020, 49, 20190265. [CrossRef] [PubMed]

12. Sklavos, A.; Delpachitra, S.; Jaunay, T.; Kumar, R.; Chandu, A. Degree of Compression of the Inferior Alveolar Canal on ConeBeam Computed Tomography and Outcomes of Postoperative Nerve Injury in Mandibular Third Molar Surgery. J. Oral Maxillofac. Surg. 2021, 79, 974-980. [CrossRef]

13. Alsufyani, N.A.; Flores-Mir, C.; Major, P.W. Three-dimensional segmentation of the upper airway using cone beam CT: A systematic review. Dentomaxillofac. Radiol. 2012, 41, 276-284. [CrossRef] [PubMed] 
14. Burström, G.; Buerger, C.; Hoppenbrouwers, J.; Nachabe, R.; Lorenz, C.; Babic, D.; Homan, R.; Racadio, J.M.; Grass, M.; Persson, O.; et al. Machine learning for automated 3-dimensional segmentation of the spine and suggested placement of pedicle screws based on intraoperative cone-beam computer tomography. J. Neurosurg. Spine 2019, 31, 147-154. [CrossRef] [PubMed]

15. Neelapu, B.C.; Kharbanda, O.P.; Sardana, V.; Gupta, A.; Vasamsetti, S.; Balachandran, R.; Rana, S.S.; Sardana, H.K. A pilot study for segmentation of pharyngeal and sino-nasal airway subregions by automatic contour initialization. Int. J. Comput. Assist. Radiol. Surg. 2017, 12, 1877-1893. [CrossRef] [PubMed]

16. Galibourg, A.; Dumoncel, J.; Telmon, N.; Calvet, A.; Michetti, J.; Maret, D. Assessment of automatic segmentation of teeth using a watershed-based method. Dentomaxillofac. Radiol. 2018, 47, 20170220. [CrossRef]

17. Kauke, M.; Safi, A.F.; Kreppel, M.; Grandoch, A.; Nickenig, H.J.; Zöller, J.E.; Dreiseidler, T. Size distribution and clinicoradiological signs of aggressiveness in odontogenic myxoma-three-dimensional analysis and systematic review Dentomaxillofac. Radiol. 2018, 47, 20170262. [CrossRef]

18. Yushkevich, P.A.; Piven, J.; Hazlett, H.C.; Smith, R.G.; Ho, S.; Gee, J.C.; Gerig, G. User-guided 3D active contour segmentation of anatomical structures: Significantly improved efficiency and reliability. NeuroImage 2006, 31, 1116-1128. [CrossRef] [PubMed]

19. Botticelli, S.; Verna, C.; Cattaneo, P.M.; Heidmann, J.; Melsen, B. Two- versus three-dimensional imaging in subjects with unerupted maxillary canines. Eur. J. Orthod. 2011, 33, 344-349. [CrossRef] [PubMed]

20. Michetti, J.; Maret, D.; Mallet, J.P.; Diemer, F. Validation of cone beam computed tomography as a tool to explore root canal anatomy. J. Endod. 2010, 36, 1187-1190. [CrossRef] [PubMed]

21. Li, Y.; Qiao, S.C.; Gu, Y.X.; Zhang, X.M.; Shi, J.Y.; Lai, H.C. A novel semiautomatic segmentation protocol to evaluate guided bone regeneration outcomes: A pilot randomized, controlled clinical trial. Clin. Oral Implant. Res. 2019, 30, 344-352. [CrossRef] [PubMed]

22. Antila, K.; Lilja, M.; Kalke, M. Segmentation of facial bone surfaces by patch growing from cone beam CT volumes. Dentomaxillofac. Radiol. 2016, 45, 20150435. [CrossRef] [PubMed]

23. Abdolali, F.; Zoroofi, R.A.; Otake, Y.; Sato, Y. A novel image-based retrieval system for characterization of maxillofacial lesions in cone beam CT images. Int. J. Comput. Assist. Radiol. Surg. 2019, 14, 785-796. [CrossRef] [PubMed]

24. Koerich, L.; Burns, D.; Weissheimer, A.; Claus, J.D.P. Three-dimensional maxillary and mandibular regional superimposition using cone beam computed tomography: A validation study. Int. J. Oral Maxillofac. Surg. 2016, 45, 662-669. [CrossRef] [PubMed]

25. Shaheen, E.; Khalil, W.; Ezeldeen, M.; Van de Casteele, E.; Sun, Y.; Politis, C.; Jacobs, R. Accuracy of segmentation of tooth structures using 3 different CBCT machines. Oral Surg. Oral Med. Oral Pathol. Oral Radiol. 2017, 123, 123-128. [CrossRef] [PubMed]

26. Agbaje, J.O.; de Casteele, E.V.; Salem, A.S.; Anumendem, D.; Lambrichts, I.; Politis, C. Tracking of the inferior alveolar nerve: Its implication in surgical planning. Clin. Oral Investig. 2017, 21, 2213-2220. [CrossRef]

27. Vallaeys, K.; Kacem, A.; Legoux, H.; Le Tenier, M.; Hamitouche, C.; Arbab-Chirani, R.1. 3D dento-maxillary osteolytic lesion and active contour segmentation pilot study in CBCT: Semiautomatic vs manual methods. Dentomaxillofac. Radiol. 2015, 44, 20150079. [CrossRef] [PubMed] 